\title{
Performance of an Automated Real-time ST-Segment Analysis Program to Detect Coronary Occlusion and Reperfusion
}

\author{
Rolf F. Veldkamp, MD, PhD, Sharon Sawchak, RN, James E. Pope, MD, \\ Robert M. Califf, MD, and Mitchell W. Krucoff, MD
}

\begin{abstract}
Continuously updated ST-segment recovery analysis has been shown to accurately predict infarct-related artery patency. Salient principles were converted into algorithms and incorporated into a portable ST monitor for optimal application. This study tested the automated program's ability to detect occlusion and reperfusion during balloon angioplasty. ST-segment recordings during 78 balloon occlusions in 31 patients were analyzed. The program requires at least one electrocardiogram with ST elevation of $200 \mu \mathrm{V}$ or greater in the recording, caused by the current occlusion or by a previous occlusion, before it will yield a patency prediction. All 35 inflations causing peak ST elevation of $200 \mu \mathrm{V}$ or more were indeed detected. All five inflations causing less than $200 \mu \mathrm{V}$ ST elevation preceded by an inflation causing 200 $\mu V$ or higher ST elevation were also detected. Occlusion was detected a median of 40 seconds after inflation, and reperfusion a median of 17 seconds after deflation. Peak ST elevation greater than $200 \mu \mathrm{V}$ occurred in 19 of 26 left anterior descending artery inflations ( $73 \%), 1$ of 22 left circumflex artery LCX inflations $(5 \%)$, and 15 of 30 right coronary artery inflations $(50 \%)$. Five different leads identified peak ST elevation through 12-lead surveillance. In this model of coronary occlusion during angioplasty balloon inflation, the automated patency assessment program appears to detect coronary angioplasty balloon occlusion and reperfusion within seconds in all occlusions causing a peak ST elevation of $200 \mu \mathrm{V}$ or greater. Testing this automated patency assessment program as a noninvasive triage tool in myocardial infarction patients seems warranted. Key words: automated ST-segment analysis, balloon angioplasty, coronary occlusion, reperfusion, patency assessment, acute myocardial infarction.
\end{abstract}

Patients suffering acute myocardial infarction in whom thrombolytic therapy fails to provide early and stable reperfusion might benefit from addi-

From the Division of Cardiology, Department of Medicine, Duke University Medical Center, Durham, North Carolina.

Reprint requests: Rolf F. Veldkamp, MD, PhD, Academic Hospital "Dijkzigt," Thorax Center Bd 434, Eramus University Rotterdam, Postbus 1738, 3000 DR Rotterdam, The Netherlands. tional pharmacologic or mechanical revascularization therapy (1-9). To study these patients without subjecting all patients to catheterization requires a practical, accurate, noninvasive method that is able to detect both reperfusion and reocclusion in real time.

Analysis of ST-segment recovery has been shown to accurately predict infarct artery patency in a real-time emulation using dynamically updated 
reference measurements during continuous digital 12-lead ST-segment monitoring $(10-12)$. The use of updated reference electrocardiograms (ECGs) for comparison of ST measurements rather than static pre- and post-thrombolysis ECGs may result in more adequate prediction of patency when multiple ST recovery and reelevation episodes occur in the early phase of acute myocardial infarction (10-12). However, this continuous ST monitoring method of patency assessment requires transfer of the ECG data to a separate personal computer review station with software providing a threedimensional graphic display of ST deviation over time in all 12 leads, as well as superimposed comparison of single ECGs in full disclosure with measurements of ST deviation in each of the leads (ST3D, Tampa Cardiovascular Associates, Tampa, FL) (10). The procedures for performing a patency assessment with this custom software include manual identification of peaks and troughs in the ST trendline over time, requiring both time and specific training of the clinician. To enhance bedside application, the ST-segment recovery analysis should be a component of the free-standing monitor itself and should be accessible to less intensively trained physicians. Salient principles of the analysis method were therefore converted into algorithms and incorporated into the portable monitor as an automated patency assessment program (13). This study was designed as a first phase of testing the ability of this automated patency assessment system to detect known periods of coronary occlusion and reperfusion and the speed thereof, using angioplasty as a controlled human model.

\section{Materials and Methods}

\section{Patient Population}

Patients undergoing elective percutaneous transluminal coronary angioplasty of one or more subtotal $(75-99 \%)$ stenotic lesions were selected prospectively so that equal numbers of patients were included in three groups representing each of the three major coronary arteries. Patients were excluded if they had a history of coronary artery bypass operation or an acute myocardial infarction within 3 days before the angioplasty procedure. Patients who had a chronic total occlusion as a target lesion, those with an intraventricular conduction delay, and those undergoing an intervention with a device other than a conventional balloon were also excluded. Coronary angioplasty and ST- segment monitoring were performed according to standard practice in our institution.

\section{ST-Segment Monitoring}

The 12-lead ST monitor (ST100, Mortara Instrument, Milwaukee, WI) has been described in detail previously (14-16). In summary, the ST monitor acquires and digitizes a standard 12-lead ECG every 20 seconds, recognizing QRS onset in all 12 leads simultaneously and creating a median beat complex out of each acquisition. The ST-segment measurements were performed by the ST monitor $60 \mathrm{~ms}$ after the $\mathrm{J}$ point. For the purpose of this study, all acquired ECGs were stored in the monitor's memory. Torso lead placement (15) with radiotranslucent electrodes and impedance-regulated skin preparation (Quik Prep, Quinton Medical, Seattle, WA) were used to reduce noise.

\section{Automated Patency Assessment}

Details of the automated patency assessment method have been described previously (13). Briefly, continuously updated surveillance of all 12 leads is used to select the most active lead, defined as the single lead showing the highest ST-segment elevation up to that moment. Amplitude measurements in this most active lead are then used to identify two ECGs, the last transition ECG and the maximum ECG, for comparison with the assessment ECG. As shown in Figure 1, the last transition ECG is defined as the last ECG prior to the assessment ECG at which the trend of ST-segment amplitude over time changes from worsening (upsloping trend) to improvement (downsloping trend) or vice versa. Thus, the last transition ECG represents the last turning point in the trendline of ST amplitudes over time (13). The maximum ECG is defined as the ECG displaying the greatest STsegment elevation in the most active lead during the recording episode up to the moment of assessment. With each new assessment ECG, the most active lead and the ECGs representing the maximum ECG and the last transition ECG are automatically redefined.

Analog waveforms from all three ECGs (assessment, maximum, last transition) are printed in hard copy as a comparison plot for inspection by the clinician. By pushing a button, the clinician may reject any ECG that contains unacceptable noise or conduction disturbance, after which the program selects a new ECG. After visual confirma- 


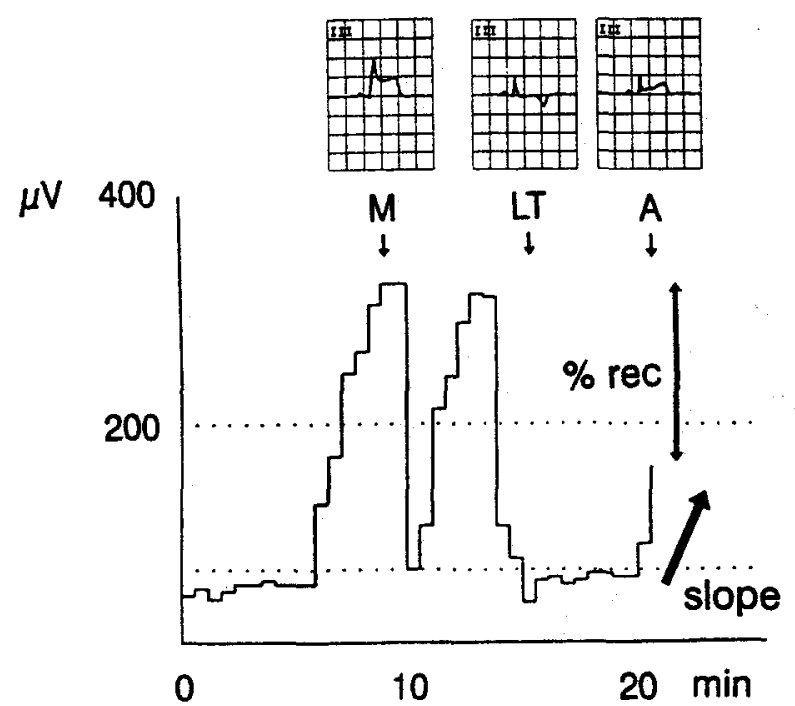

Fig. 1. ST-segment deviation over time measured in the peak lead (III) during three consecutive balloon inflations. The definition of the maximum ECG (M), last transition ECG (LT), and assessment ECG (A) are represented above the trendline. Two arrows on the right side represent the two measurements that are used in a logic flow to come to an interpretative statement regarding patency of the coronary artery: the recovery in ST elevation in the assessment ECG as a percentage of the maximum ST elevation ( $\%$ rec); and the 2 -minute peak slope in the trendline between the last transition ECG and the assessment ECG.

tion of satisfactory noise levels, two parameters of ST recovery are automatically calculated: (1) the ST amplitude recovery in the assessment ECG as a percentage of the maximum ECG ST amplitude; and (2) the peak 2-minute ST trend slope between the last transition ECG and the assessment ECG (Fig. 1). A window between two points 2 minutes apart is used "floating" backward from the assessment ECG to determine the slope between these two points at each 20 -second interval: worsening (upsloping $\geq 50 \mu \mathrm{V}$ in 2 minutes), improvement (downsloping $\leq-50 \mu \mathrm{V}$ in 2 minutes), or flat. The last transition ECG is the last ECG prior to the assessment ECG at which the trend of ST amplitudes over time in the worst lead changes from upsloping to downsloping or vice versa (Fig. 1).

As previously described (13), the results of these two calculations are then conveyed through a simple logic flow (Fig. 2) to produce a final interpretative statement about coronary patency as occluded, indeterminate, or patent. If less than $50 \%$ recovery has occurred and the peak 2-minute ST slope is flat or upsloping, the infarct-related artery is consid-

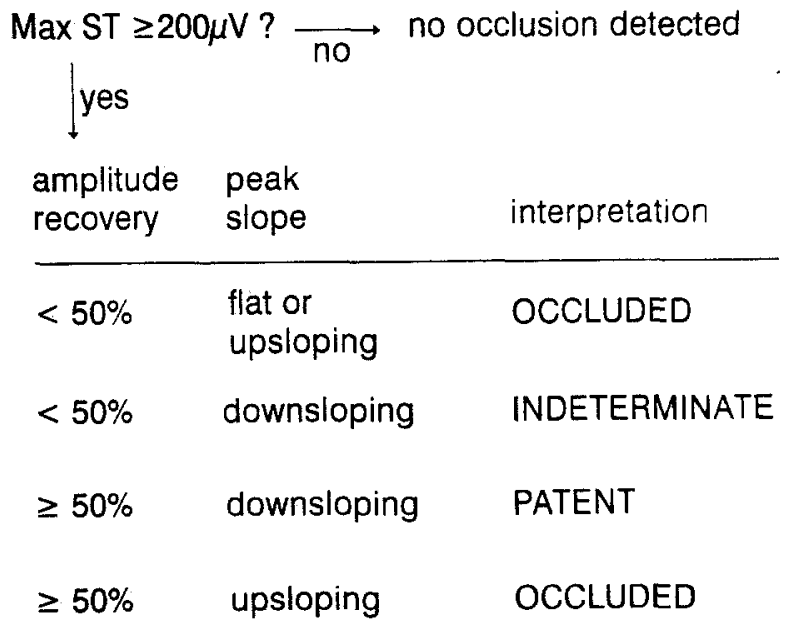

Fig. 2. Representation of the logic flow implemented in the automated patency assessment program. The two measurements demonstrated in Figure 1 are used to come to a final statement regarding infarct-related artery patency. Modified from Veldkamp et al. (13). With permission.

ered to have remained occluded. A recovery of $50 \%$ or more from the peak ECG, as well as a downsloping peak 2-minute ST slope, is required to assess the infarct-related artery as patent in order to differentiate it from gradual amplitude diminution due to loss of myocardial mass producing injury current. If a recovery of less than $50 \%$ has occurred but the peak ST slope is downsloping, it is believed, but without enough certainty, that this might indicate the advent of reperfusion, and the patency assessment is therefore indeterminate. A repeat patency assessment should be made a few minutes later to see whether ST resolution has indeed proceeded. When $50 \%$ recovery has occurred with the required downsloping peak 2minute ST slope but is then followed by an upsloping ST trend, it is believed that this is due to recurrent occlusion of the infarct-related artery, and this leads to assessment of the artery as occluded.

If none of the recorded ECGs has an ST amplitude of $200 \mu \mathrm{V}$ or greater, a special statement is printed to the effect that the amplitudes are below the programmed threshold to perform a patency assessment. Thus, only if the current occlusion or a previous occlusion caused an ST amplitude of 200 $\mu V$ or greater in the selected lead will the algorithm perform a patency assessment. This minimum threshold was chosen because the accuracy of STsegment recovery analysis may be reduced in patients with lower ST amplitudes during their 
acute myocardial infarction (data from the GUSTO1 ECG monitoring substudy).

\section{Endpoints}

For the purpose of this study, a patency assessment was performed on each ECG recorded every 20 seconds before, during, and after each balloon inflation lasting at least 60 seconds. A ballooninduced occlusion was considered detected if the program produced at least one interpretative statement saying "occluded" during that inflation. For all detected balloon inflations, the following performance parameters were evaluated: (1) time from balloon inflation to the first assessment ECG with an interpretative statement saying "occluded"; (2) time from balloon deflation to the first assessment ECG with an interpretative statement no longer saying "occluded"; and (3) duration of the episode during which interpretative statements said "occluded" as compared with the actual duration of balloon inflation.

\section{Statistics}

All continuous variables are represented as median values, 25 th -75 th percentiles, and in relevant cases as ranges. Categorical parameters are represented as numbers of patients or number of inflations. Detection rates are reported as absolute numbers, percentages, and $95 \%$ confidence limits. Differences in detection rates over all inflations were tested by Fisher's exact test; $P$ values $<.05$ were considered significant.

\section{Results}

A total of 78 transient coronary occlusions were recorded from 31 coronary sites in 30 patients. The recordings of six patients were excluded from analysis: two underwent percutaneous transluminal coronary angioplasty of a totally occluded artery, two underwent angioplasty with a perfusion balloon catheter, one had continuous right bundle branch block, and one had incomplete left bundle branch block with axis changes during inflation. Table 1 summarizes some of the characteristics of the study population. The vessel dilated was the left anterior descending artery (LAD) in 10 patients (26 inflations), the left circumflex artery (LCX) in 10 patients (22 inflations), and the right coronary artery (RCA) in II patients (30 inflations). Median duration of inflation was $183 \mathrm{sec}-$ onds (quartile range, 126-189 seconds; range, 62-912 seconds). Median peak ST deviation per inflation was $165 \mu \mathrm{V}$ (quartile range, 34-258 $\mu \mathrm{V}$; range, $-190-968 \mu \mathrm{V})$. Median peak ST deviation was $240 \mu \mathrm{V}$ for LAD inflations (quartile range, $115-303 \mu \mathrm{V}$; range, $65-968 \mu \mathrm{V}) ;-20 \mu \mathrm{V}$ for LCX inflations (quartile range, -95-15 $\mu \mathrm{V}$; range, $-190-820 \mu \mathrm{V})$; and $205 \mu \mathrm{V}$ for RCA inflations (quartile range, $78-275 \mu \mathrm{V}$; range, $-20-395 \mu \mathrm{V}$ ). Median peak ST deviation was $175 \mu \mathrm{V}$ for first balloon inflations (quartile range, 15-235 $\mu \mathrm{V}$; range, -115 to $687 \mu \mathrm{V}$ ) and $160 \mu \mathrm{V}$ for repeated balloon inflations (quartile range, $50-260 \mu \mathrm{V}$; range, $-190-968 \mu \mathrm{V})$. Of the 78 inflations, there were 35 $(45 \%)$ causing peak ST amplitude at $200 \mu \mathrm{V}$ or more, including 19 of $26 \mathrm{LAD}$ inflations $(73 \%), 1$ of 22 LCX inflations ( $5 \%$ ), and 15 of 30 RCA infla-

Table 1. Patient Characteristics

\begin{tabular}{ll}
\hline Sex & $15 / 30(50 \%)$ \\
$M$ & $15 / 30(50 \%)$ \\
$F$ & Median, 68) (quartile range, 51-75; range, 40-84) \\
Age (years) & $18 / 30(60 \%)$ \\
Hypertension & $12 / 30(40 \%)$ \\
Diabetes mellitus & \\
Previous AMI same region & $5 / 30(17 \%)$ \\
$\quad$ Non-Q wave & $5 / 30(17 \%)$ \\
$\quad$ Q wave & $15 / 30(50 \%)$ \\
Indication & $10 / 30(33 \%)$ \\
$\quad$ Angina pectoris & $4 / 30(13 \%)$ \\
$\quad$ Unstable angina & $1 / 30(3 \%)$ \\
$\quad$ Post-AMI & $14 / 30(47 \%)$ \\
$\quad$ Syncope & $8 / 30(27 \%)$ \\
Extent of disease & $8 / 30(27 \%)$ \\
$\quad$ vessel & Median, $95($ quartile range, 75-95; range, 75-99) \\
$\quad$ 2 vessels &
\end{tabular}

AMI, acute myocardial infarction; post-AMI, $>3$ days after acute myocardial infarction. 
tions $(50 \%)$. All 35 inflations ( $100 \%$ ) with $200 \mu \mathrm{V}$ or greater peak ST amplitude were detected. Of the 43 balloon inflations that caused less than $200 \mu \mathrm{V}$ ST elevation, 5 were also detected (1 LAD, 1 LCX, 3 RCA). In all five instances, the inflation followed a prior inflation that did cause a $200 \mu \mathrm{V}$ or greater ST elevation, in which case the automated patency assessment program was allowed to produce a patency assessment statement.

In the 40 detected inflations, median time from balloon inflation to detection of occlusion was 40 seconds (quartile range, 20-80 seconds; range, $0-120$ seconds). Reperfusion by balloon deflation in those same 40 occlusions was detected at a median of 17 seconds (quartile range, $-2-16$ seconds; range, $-92-126$ seconds). The median duration of occlusion defined by the automated patency assessment program was 120 seconds of the actual median balloon inflation duration of 181 seconds in these 40 detected inflations. During six inflations, one or more ECGs were not analyzed owing to conduction changes (one, two, two, three, three, and four consecutive ECGs excluded, respectively) and one ECG during one inflation was not analyzed owing to the high noise content.

After splitting the 78 balloon inflations around the median duration of inflation, no correlation was found between inflation duration and the program's detection performance $(P=1.0)$. Neither was there any difference in detection of first versus repeated balloon inflations: 15 of 30 versus 25 of 48 , respectively $(P=1.0)$.

The precordial location of the peak lead activity varied with the artery occluded. Of the standard 12 leads monitored, the following peak leads were used to detect occlusions: leads $V_{1}(2), V_{2}(8), V_{3}$ (8), and $\mathrm{V}_{4}$ (2) for LAD occlusions; lead III (2) for LCX occlusions; and lead III (18) for RCA occlusions. Thus, detection of the occlusions occurred in five different leads.

\section{Discussion}

The results of this study show that this automated patency assessment program wholly contained within the ST monitor's architecture detected all occlusions producing injury current of $200 \mu \mathrm{V}$ or greater, equivalent to entry criteria used in some thrombolytic trials. Detection of multiple episodes of occlusion and reperfusion was within seconds of their actual occurrence. Longer inflations did not increase the chance of detection in this model. This is probably due to our standard angioplasty practice of deflating the balloon earlier if ischemia induced by the occlusion is relatively severe, while dilating for longer periods if the patient tolerates it well with no ischemia. In spontaneous occlusion and infarction, longer acute occlusions would be expected to produce higher ST-segment amplitudes. Repeated inflations were detected as well as first inflations. Although one might expect lower amplitudes in repeated balloon inflations, this was not the case in this population. Furthermore, after an initial occlusion causing a $200 \mu \mathrm{V}$ or higher ST elevation, the program relies on ST trend slopes and amplitudes relative to the peak ST elevation rather than on absolute amplitudes.

It is notable that the peak lead selected varied over the precordium with the artery occluded. This suggests that automated detection of coronary occlusion not only is a matter of updated assessments of ST amplitudes, but also is dependent on the ability to select a monitoring lead central to the geographic pattern of ST deviation (17-21).

\section{Limitations}

Elective coronary angioplasty is an imperfect model to test a program designed for acute myocardial infarction applications. It precludes an analysis of the sensitivity and specificity of automated occlusion detection because detection of coronary occlusion while the balloon is deflated may signify other "true" occlusion causes, such as coronary occlusion by the guiding catheter or rapid thrombotic occlusion due to dissection of the arterial wall. Even injection of contrast solution into the coronary artery may result in temporary ST-segment shifts. The fact that only 40 of the 78 balloon inflations produced enough injury current to fulfill the preset requirement of $200 \mu \mathrm{V}$ illustrates another reason why coronary angioplasty is an imperfect model for coronary occlusion in acute myocardial infarction. On the basis of the entry criteria used for some thrombolytic trials, such ST levels are somewhat more common in the milieu of prolonged occlusion necrosis typical of actual acute myocardial infarction. In a standard 12-lead ECG format, such criteria have been demonstrated to promote an anatomic bias against LCX occlusions (17), which is unfortunately paralleled in these data taken during coronary angioplasty, with markedly lower peak ST elevation during occlusions of the LCX. In this regard, the percutaneous transluminal coronary angioplasty model emulated acute myocardial infarctions well, with most LCX balloon occlusions failing to produce the minimum 
ST-segment elevation required by the automated patency program.

Despite these limitations, coronary angioplasty provides a human model of coronary occlusion and reperfusion of known duration and location, which is very useful for preliminary testing of a prototype patency assessment program. Every recorded ECG could thus be correlated with simultaneous angiographic documentation of the location and flow status of the artery. The previous work on which this program was modeled has demonstrated that during infarction the myocardium generates and resolves ST-segment shift abruptly enough to make continuous ST recovery analysis a usefully accurate noninvasive marker of patency $(10-12)$. The results of this study suggest that further investigation into the performance of the automated patency assessment program during actual acute myocardial infarction is warranted.

The time course of ST-segment change during acute myocardial infarction may be substantially different from trends obtained during coronary angioplasty and might be more heterogeneous. Whether the anatomic bias or the overall performance of the program could be further optimized by changing minimal amplitude requirements or slope rates is beyond the scope of this balloon angioplasty model and needs further investigation in the acute myocardial infarction setting. Use of additional leads or modified lead systems might also enhance performance $(20,21)$, especially to reduce the anatomic bias against LCX occlusions, although the 12-lead system is practical because of universal familiarity. The variety of leads used to detect coronary occlusion suggest that more restricted lead systems would risk decreasing the accuracy of the algorithm. They would also diminish the ability to compare the precordial multilead "fingerprint" patterns that are useful in discriminating reocclusion of the culprit lesion from other sources of ST-segment deviation (17-20). Other modifications to reduce the anatomic bias and increase the program's sensitivity, such as variable thresholds, use of reciprocal ST depression, or coronary artery-specific algorithms may also bear exploration.

\section{Clinical Implications}

Within the controlled setting of coronary angioplasty, the automated patency assessment program appears to be capable of noninvasive detection of coronary occlusion and reperfusion within seconds of their occurrence. Contained entirely within the architecture of a portable bedside ST monitor, this automated patency assessment program might become a practical and valuable tool in the management of acute myocardial infarction patients following thrombolytic therapy. A large clinical field trial testing the program during actual acute myocardial infarction is currently ongoing.

\section{Acknowledgments}

The authors are greatly indebted to A. Karen Loeffler and Suzanne E. Crater for their effort in gathering the data. They thank the staff and personnel of the Interventional Coronary Catheterization laboratory for their help in this project and also thank Galen S. Wagner for carefully and critically reviewing the manuscript.

\section{References}

1. Simoons ML, Serruys PW, van den Brand M et al: Early thrombolysis in acute myocardial infarction: limitation of infarct size and improved survival. J Am Coll Cardiol 7:717, 1986

2. Vermeer F, Simoons ML, Bär FW et al: Which patients benefit most from early thrombolytic therapy with intracoronary streptokinase? Circulation 74:1379, 1986

3. Ohman EM, Califf RM, Topol EJ et al: Consequences of reocclusion after successful reperfusion therapy in acute myocardial infarction. Circulation 82:781, 1990

4. Califf RM, Topol EJ, George BS et al: Characteristics and outcome of patients in whom reperfusion with intravenous tissue-type plasminogen activator fails: results of the thrombolysis and angioplasty in myocardial infarction (TAMI) I trial. Circulation 77 : 1090,1988

5. White HD, Norris RM, Brown MA et al: Effect of intravenous streptokinase on left ventricular function and early survival after acute myocardial infarction. N Engl J Med 317: 850, 1987

6. Belenkie I, Traboulsi M, Hall CA et al: Rescue angioplasty during myocardial infarction has a beneficial effect on mortality: a tenable hypothesis. Can J Cardiol 8:357, 1992

7. Ellis SG, Ribeiro da Silva E, Heyndrickx G et al: Randomized comparison of rescue angioplasty with conservative management of patients with early failure of thrombolysis for acute myocardial infarction. Circulation 90:2280, 1994

8. The GUSTO Angiographic Investigators: The effects of tissue plasminogen activator, streptokinase, or both on coronary-artery patency, ventricular function, and survival after acute myocardial infarction. N Engl J Med 329:1615, 1993 
9. Lenderink T, Simoons ML, Van Es GA et al: Benefit of thrombolytic therapy is sustained throughout five years, and is related to TIMI perfusion grade 3 but not grade 2 flow at discharge. Circulation 92:110, 1995

10. Krucoff MW, Croll MA, Pope JE et al: Continuously updated 12-lead ST-segment recovery analysis for myocardial infarct artery patency assessment and its correlation with multiple simultaneous early angiographic observations. Am J Cardiol 71:145, 1993

11. Krucoff MW, Croll MA, Pope JE et al: Accuracy of a "real-time" oriented non-invasive method for the detection of failed reperfusion using continuous 12lead ST segment recovery analysis. Circulation 88: 437, 1993

12. Veldkamp RF, Green CL, Wilkins ML et al: Comparison of continuous ST-segment recovery analysis with methods using static electrocardiograms for noninvasive patency assessment during acute myocardial infarction. Am J Cardiol 73:1069, 1994

13. Veldkamp RF, Bengtson JR, Sawchak ST et al: Evolution of an automated ST-segment analysis program for dynamic real-time, noninvasive detection of coronary occlusion and reperfusion. J Electrocardiol 25(suppl):182, 1993

14. Krucoff MW, Croll MA, Pope JE et al: Heuristic and logistic principles of ST-segment interpretation in the time domain: evolution in the context of the TAMI-7 trial design. J Electrocardiol 23(suppl):6, 1989

15. Krucoff MW, Wagner NB, Pope JE et al: The portable programmable microprocessor driven real-time 12 - lead electrocardiographic monitor: a preliminary report of a new device for the noninvasive detection of successful reperfusion or silent coronary reocclusion. Am J Cardiol 65:143, 1990

16. Adams IM, Mortara DW: A new method for electrocardiographic monitoring. In Califf RM, Wagner GS (eds): Acute coronary care. Martinus Nijhof, Boston, 1987

17. Saetre HA, Selvester RH, Solomon JC et al: 16 Lead ECG changes with coronary angioplasty: location of ST-T changes with balloon occlusion of five arterial perfusion beds. J Electrocardiol 24(suppl):153, 1992

18. Krucoff MW, Pope JE, Bottner RK et al: Computerassisted ST-segment monitoring: experience during and after brief coronary occlusion. J Electrocardiol 20 (suppl):15, 1987

19. Krucoff MW, Parente AR, Bottner RK et al: Stability of multilead ST-segment "fingerprints" over time after percutaneous transluminal coronary angioplasty and its usefulness in detecting reocclusion. Am J Cardiol 61:1232, 1988

20. Bush HS, Ferguson JJ III, Angelini P, Willerson JT: Twelve-lead electrocardiographic evaluation of ischemia during percutaneous transluminal coronary angioplasty and its correlation with acute reocclusion. Am Heart J 121:1591, 1991

21. Kornreich F, Montague TJ, Rautaharju PM: Body surface potential mapping of ST segment changes in acute myocardial infarction. Implications for ECG enrollment criteria for thrombolytic therapy. Circulation 87:773, 1993 